\title{
The evolutionary state of Miras with changing pulsation periods ${ }^{\star} \star \star \star$
}

\author{
S. Uttenthaler ${ }^{1,2}$, K. Van Stiphout ${ }^{1,3}$, K. Voet $^{1}$, H. Van Winckel $^{1}$, S. Van Eck ${ }^{4}$, A. Jorissen ${ }^{4}$, F. Kerschbaum ${ }^{2}$, \\ G. Raskin ${ }^{1}$, S. Prins ${ }^{1}$, W. Pessemier ${ }^{1}$, C. Waelkens ${ }^{1}$, Y. Frémat ${ }^{5}$, H. Hensberge ${ }^{5}$, L. Dumortier ${ }^{5}$, and H. Lehmann ${ }^{6}$ \\ ${ }^{1}$ Instituut voor Sterrenkunde, University of Leuven, Celestijnenlaan 200D, 3001 Leuven, Belgium \\ e-mail: stefan@ster. kuleuven . be \\ 2 University of Vienna, Department of Astronomy, Türkenschanzstraße 17, 1180 Vienna, Austria \\ 3 Instituut voor Kern- en Stralingsfysica, University of Leuven, Celestijnenlaan 200D, 3001 Leuven, Belgium \\ ${ }^{4}$ Institut d'Astronomie et d'Astrophysique, Université Libre de Bruxelles, CP226, Boulevard de Triomphe, 1050 Brussels, Belgium \\ 5 Royal Observatory of Belgium, Ringlaan 3, 1180 Brussels, Belgium \\ 6 Thüringer Landessternwarte Tautenburg, Sternwarte 5, 07778 Tautenburg, Germany
}

Received 7 January 2011 / Accepted 17 April 2011

\section{ABSTRACT}

Context. Miras are long-period variables thought to be in the asymptotic giant branch (AGB) phase of evolution. In about one percent of known Miras, the pulsation period is changing. It has been speculated that this changing period is the consequence of a recent thermal pulse in these stars.

Aims. We aim to clarify the evolutionary state of these stars, and to determine in particular whether or not they are in the thermallypulsing (TP-)AGB phase.

Methods. One important piece of information that has been neglected so far when determining the evolutionary state is the presence of the radio-active $s$-process element technetium (Tc). We obtained high-resolution, high signal-to-noise-ratio optical spectra of a dozen prominent Mira variables with changing pulsation period to search for this indicator of TPs and dredge-up. We also use the spectra to measure lithium (Li) abundances. Furthermore, we establish the evolutionary states of our sample stars by means of their present-day periods and luminosities.

Results. Among the twelve sample stars observed in this programme, five were found to show absorption lines of Tc. BH Cru is found to be a carbon-star, its period increase in the past decades possibly having stopped by now. We report a possible switch in the pulsation mode of T UMi from Mira-like to semi-regular variability in the past two years. R Nor, on the other hand, is probably a fairly massive AGB star, which could be true for all meandering Miras. Finally, we assign RU Vul to the metal-poor thick disk with properties very similar to the short-period, metal-poor Miras.

Conclusions. We conclude that there is no clear correlation between period change class and Tc presence. The stars that are most likely to have experienced a recent TP are BH Cru and R Hya, although their rates of period change are quite different.

Key words. stars: AGB and post-AGB - stars: oscillations - stars: evolution - stars: abundances

\section{Introduction}

Mira-type variables are red giant stars that exhibit long-period variability with large amplitude $(\Delta V>2 \mathrm{~m}$.5). The pulsation period of most Mira variables is stable over long time spans (i.e. of between decades and centuries), but there are exceptions. Zijlstra \& Bedding (2002) define three classes of secular period change in Mira variables:

Continuous change: a continuous decrease or increase in the period over a century or more, with no indication for epochs of a stable period. The change in period is in all cases large, $15 \%$ or more over 100 years. In the following, we refer to this class as $\mathrm{CCh}$.

\footnotetext{
* Based on observations made with the Mercator Telescope, operated on the island of La Palma by the Flemish Community, at the Spanish Observatorio del Roque de los Muchachos of the Instituto de Astrofísica de Canarias, and with the Swiss $1.2 \mathrm{~m}$ Euler telescope at La Silla, Chile. $\star \star$ The spectra are only available at the CDS via anonymous ftp to cdsarc.u-strasbg.fr $(130.79 .128 .5)$ or via http://cdsarc.u-strasbg.fr/viz-bin/qcat?]/A+A/531/A88
}

Sudden change: after a long phase of stability, the period suddenly begins to either decrease or increase at a large rate. The total change in period is similar to that of the CCh class, but the rate is ten times faster because the same change is achieved within a decade rather than within a century (SCh in the following, not to be confused with the SC spectral type!).

Meandering change: a number of long-period Miras show evidence of meandering or fluctuating periods. The periods change by $10 \%$ over several decades, followed by a return to the previous period. The rate of change is comparable to that of the CCh Miras, but the total change is somewhat smaller than that of the other two classes. Most Miras in this class have periods in excess of $400 \mathrm{~d}$ (MCh in the following).

Templeton et al. (2005) study the period evolution of 547 Mira variables, of which 57 are found to have a period change at the $2 \sigma$ confidence level, and eight stars at the level of $6 \sigma$. Their catalogue is the current reference for observed secular evolution in Miras in the solar neighbourhood. 
From the point of view of stellar evolution, Mira variables are thought to be in the asymptotic giant branch (AGB) phase. The hydrogen-burning shell is the main energy source during most of the time on the AGB, which is quasi-periodically interrupted by powerful ignitions of the helium-burning shell (Schwarzschild \& Härm 1965). These ignitions are called helium-shell flashes or thermal pulses (TPs), hence this phase is also referred to as the TP-AGB. A TP has a large impact on the structure of the whole star: the luminosity, temperature, radius, and thus pulsation period of the outer envelope, are predicted to vary strongly within centuries. For a star with $2 M_{\odot}$ in the middle of its TP-AGB phase, the TP itself (convective zone in the intershell region) lasts about 300-400 years. The evolution during the TP is governed by the thermal timescale of the envelope, and the evolution is faster in both later TPs and more massive stars. Several hundred years after the onset of the TP, surface abundance changes can occur as the third dredge-up (3DUP) begins to operate. This mixing event may take place after a TP when the convective envelope deepens to reach layers that have previously undergone nuclear processing (Busso et al. 1999). The onset of 3DUP is predicted to coincide with a temporary luminosity maximum. Once the convective envelope reaches layers with processed material, it should become visible on the stellar surface very quickly because the turn-over time of the convective envelope is of the order of only a few years.

The first suggestion that the observed period change in a few Mira stars could be related to a recent TP dates back to Wood (1975). Wood \& Zarro (1981) present evolutionary models of the TP-AGB and attempt to derive constraints on the core mass and luminosity of three Miras (R Hya, R Aql, and W Dra) with a well-known, long-term period change. The luminosity evolution inferred from the observed period change of the stars is reproduced well by the predicted luminosity evolution of the models. This gives some support to the suggestion that a TP is the cause of the period change. The fraction of Miras with large period changes $(\sim 1.6 \%$; Templeton et al. 2005) agrees well with the expected ratio of pulse duration to inter-pulse time of roughly $1-2 \%$. Hence, from a statistical point of view, we expect that at any given time at least $1 \%$ of the Miras is undergoing a TP. Whether the ones that are observed to show a large period change are also the ones that currently undergo a TP is a different question, which we aim to address here.

There are, however, also alternative explanations of the observed period changes, which do not require a recent TP. Ya'ari \& Tuchman (1996) and Lebzelter \& Wood (2005) identify a feedback mechanism between the pulsation and the stellar entropy structure, which can lead to mode switching. On the other hand, Zijlstra et al. (2004) propose that a possibly chaotic feedback between molecular opacities, pulsation amplitude, and period can cause an unstable period. This is supposed to be most important for stars with a C/O ratio very close to unity (SC and CS spectral types), as small changes in the temperature in the atmosphere can cause large changes in the molecular abundances, and hence the opacities. This alternative explanation might be applicable to the period change in the MCh group, as their periods change back and forth on a much shorter timescale than can be explained by a TP.

A piece of information that has not yet been taken into account in this discussion is the presence of the radio-active element technetium (Tc) in the atmospheres of these stars. Tc is a product of the slow neutron-capture process ( $s$-process) that takes place in deep layers of AGB stars. The $s$-process products are subsequently mixed to the surface by a 3DUP event. The longest-lived Tc isotope produced by the $s$-process is ${ }^{99} \mathrm{Tc}$ with a half-life time of 210000 years, which is short compared to stellar evolution timescales before the AGB. If observed on the surface of a star, Tc is an indisputable sign of an ongoing $s$-process and 3DUP in that star, hence indicates that the star is indeed in the TP-AGB phase of evolution. Furthermore, 3DUP mixes up carbon, the product of He-burning, which can change the star from an oxygen-rich M-type to a carbon-rich C-type star.

In previous surveys of Tc in red giant stars (e.g. Little et al. 1987), only a few Miras with changing pulsation periods were investigated, and for those that had been observed the presence of Tc was often unclear. The presence of Tc would be support for the hypothesis of a recent TP that might be the cause of the observed period change. We note, however, that the absence of Tc does not exclude that TPs are going on in a given star. Nevertheless, the absence of Tc places a constraint on the strength of the TP, because only the strongest TPs on the upper AGB will be followed by a 3DUP event.

In addition to Tc, the light element lithium (Li) is an important diagnostic tool for stellar evolution studies, in particular for internal mixing processes. It is very fragile because it is burnt to helium by proton captures at temperatures exceeding $3 \times 10^{6} \mathrm{~K}$. Standard stellar evolution predicts that Li should diminish during the evolution to the giant phase, and indeed red giants are generally observed to have a low $\mathrm{Li}$ abundance compared to main sequence stars. However, standard stellar evolution also predicts that in high mass AGB stars $\left(M \gtrsim 4 M_{\odot}\right)$, Li may be produced in large amounts. In these stars, the bottom of the convective envelope extends to the $\mathrm{H}$-burning shell (also called hot bottom burning, or HBB, Sackmann \& Boothroyd 1992), which activates the Cameron-Fowler mechanism (Cameron \& Fowler 1971) producing Li. Moreover, these stars do not become C-rich by means of the dredge-up of $\mathrm{C}$, because the $\mathrm{CN}$ cycle also converts ${ }^{12} \mathrm{C}$ to ${ }^{14} \mathrm{~N}$. Li surface abundances of up to $\log \epsilon(\mathrm{Li})=4.5$ (where $\log \epsilon(\mathrm{Li})=\log N(\mathrm{Li}) / N(\mathrm{H})+12$ ) are predicted (e.g. Sackmann \& Boothroyd 1992; Karakas 2003). Luminous, Lirich stars have been observed in both the Magellanic Clouds (Smith et al. 1995) and the Galaxy (García-Hernández et al. 2007). Hence, the observation of a luminous O- and Li-rich star infers a fairly high mass for that star.

Lower-mass AGB stars can also be somewhat enriched in Li. The layer of radiative energy transport, which usually prevents the operation of the Cameron-Fowler mechanism in these stars, is naturally overcome during 3DUP events and can lead to Li enhancement at the surface up to $\log \epsilon(\mathrm{Li}) \lesssim 1.8$ (Karakas et al. 2010). In addition to this, non-convective extra-mixing processes (e.g. thermohaline mixing, magnetic buoyancy) may provide transport of matter across the radiative barrier. Observational evidence along these lines is presented by Vanture et al. (2007), Uttenthaler et al. (2007b), and Uttenthaler \& Lebzelter (2010). However, measurements of the Li abundances of O-rich, M-type AGB stars remain scarce.

For the present work, we selected several prominent representatives of the three period change classes described above and observed their optical spectra at high resolution. With these data, we can safely establish the evolutionary state of the Tc-rich stars on the TP-AGB. A central aim of the paper is to check whether the presence of $\mathrm{Tc}$ is correlated with the different period change classes. The spectra also provided us with the opportunity to measure the abundance of $\mathrm{Li}$. In addition, we determined the present-day pulsation periods of the stars from AAVSO observations, to determine whether the period change has continued in recent years. To construct an HR diagram of the stars, we calculated their luminosities with a period-magnitude relation. 
The structure of the paper is as follows. In Sect. 2, the target selection and observations are described; Sect. 3 is devoted to the analysis of the spectra and the results thereof; the results are discussed in Sect. 4, and finally Sect. 5 presents our conclusions.

\section{Target selection and observations}

The targets of our observations were selected from the stars discussed in Wood \& Zarro (1981) and Zijlstra \& Bedding (2002). They are prominent representatives of the period change classes introduced by Zijlstra \& Bedding (2002), and most are also discussed in detail in Templeton et al. (2005). In total, twelve stars were selected for the observations.

The targets that are most readily observable from the northern hemisphere were observed with the Hermes spectrograph (Raskin et al. 2010), which is mounted on the $1.2 \mathrm{~m}$ Mercator telescope at the Roque de los Muchachos observatory on the island of La Palma, Spain. The two targets best observable from the southern hemisphere were observed with the Coralie spectrograph, which is mounted on the $1.2 \mathrm{~m}$ Leonhard Euler telescope at La Silla observatory, Chile. Both Hermes and Coralie are fibre-fed, optical high-resolution spectrographs, mounted on twin telescopes. Hermes observes the range $377-900 \mathrm{~nm}$ at a spectral resolution of $R=\lambda / \Delta \lambda=85000$, whereas Coralie approximately covers the range $385-690 \mathrm{~nm}$, with a somewhat lower spectral resolution of $R=50000$. In terms of transmission, Hermes is by far the more efficient of the two instruments.

The sample stars and the log of the observations are presented in Table 1. We aimed to observe the stars close to their visual maximum brightness across their pulsation cycle, to maximise the signal-to-noise ratio $(S N R)$ of the spectra. The observed 2D spectra were reduced with the respective instrument pipeline software. As a second measure to increase the $S N R$ in the blue spectral range, we combined the individual 1D spectra into one. This was essential for some of the stars, as they have a very low flux in the blue range. Despite these efforts to increase the $S N R$, most stars have a low to moderate $S N R$ at $430 \mathrm{~nm}$. W Dra is on average the faintest star in the sample and could not be observed close to its visual brightness maximum. As a result, the $S N R$ of its spectrum in the $B$-band is essentially zero. Nevertheless, the signal in the red part of the spectrum was high enough to search for other signatures of $s$-process enrichment (see below). The $S N R$ at $671 \mathrm{~nm}$, where the Li resonance doublet lies, is about 25 in W Dra's combined spectrum, which is just good enough to determine the Li abundance. For the other stars, the $S N R$ in this spectral range is very high $(>200)$, so we used one of the individual spectra instead of a combined spectrum to measure the Li abundance (see Sect. 3.1.4).

In Table 1, the stars are grouped into the different period change classes described in the introduction, and separated by horizontal lines: The first group is that of the sudden change stars ( $\mathrm{SCh}$ ), the second one that of the continuous change stars (CCh), and the third one the meandering Miras (MCh). The star T Her in the last row is an exception here. It is a Mira variable with a constant period, hence it does not belong to any of the period change classes. However, it is discussed among other stars in Wood \& Zarro (1981), and it was hitherto uncertain whether this star contains Tc. Little et al. (1987) classify it as "Tc doubtful", and we wish to clarify this situation. Furthermore, RU Vul and W Hya are actually classified as SRVs in the Simbad database. This classification is controversial, as we will further elaborate on in Sect. 4.1.
Table 1. Observation log.

\begin{tabular}{lccrc}
\hline \hline Name & Instr. & $\begin{array}{c}\text { Date } \\
(\mathrm{dd} / \mathrm{mm} / \mathrm{yyyy})\end{array}$ & $\begin{array}{r}\text { Exp. time } \\
(\mathrm{s})\end{array}$ & $\begin{array}{c}\text { SNR } \\
\text { (at } 430 \mathrm{~nm})\end{array}$ \\
\hline BH Cru & $\mathrm{C}$ & $03 / 08 / 2009$ & $4 \times 1800$ & 12 \\
RU Vul & $\mathrm{H}$ & $04 / 07 / 2009$ & $300+2 \times 600$ & 30 \\
T UMi & $\mathrm{H}$ & $04 / 07 / 2009$ & $4 \times 1800$ & 20 \\
\hline R Aql & $\mathrm{H}$ & $04 / 07 / 2009$ & $900+2 \times 300$ & 50 \\
R Hya & $\mathrm{H}$ & $13 / 02 / 2010$ & $2 \times 400$ & 60 \\
W Dra & $\mathrm{H}$ & $15 / 08 / 2009$ & $3 \times 1800$ & $\sim 0$ \\
\hline R Nor & $\mathrm{C}$ & $17 / 08 / 2010$ & $2 \times 1800$ & 20 \\
S Her & $\mathrm{H}$ & $22 / 10 / 2009$ & $630+2 \times 1200$ & 30 \\
S Ori & $\mathrm{H}$ & $13 / 02 / 2010$ & $3 \times 1800$ & 15 \\
T Cep & $\mathrm{H}$ & $25 / 10 / 2009$ & $2 \times 1200$ & 140 \\
W Hya & $\mathrm{H}$ & $13 / 02 / 2010$ & $3 \times 150$ & 50 \\
\hline T Her & $\mathrm{H}$ & $04 / 07 / 2009$ & $2 \times 800$ & 70 \\
\hline
\end{tabular}

Notes. The meaning of the columns is as follows: (1) name listed in Simbad; (2) instrument used (H: Hermes, C: Coralie); (3) mean date of the observations; (4) exposure times of the spectra in seconds; (5) approximate $S N R$ at $430 \mathrm{~nm}$.

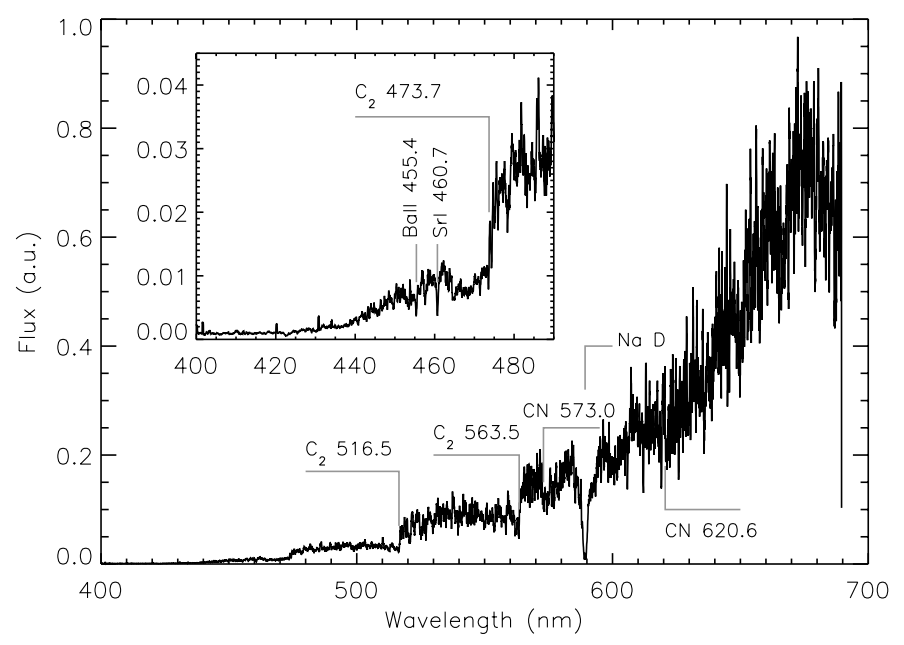

Fig. 1. Coralie spectrum of $\mathrm{BH} \mathrm{Cru}$, smoothed to a resolution of $\sim 0.3 \mathrm{~nm}$ per wavelength point. The main features of the spectrum are identified. The inset shows a zoom on the spectrum blue-ward of $490 \mathrm{~nm}$, where the flux is very low.

\section{Analysis and results}

\subsection{Analysis of the spectra}

\subsubsection{Oxygen-rich versus carbon-rich}

As a first step, we performed a global inspection of the spectra to decide whether they are dominated by either features of oxygenbearing molecules (e.g. TiO, VO) or those of carbon-bearing molecules (e.g. $\mathrm{C}_{2}, \mathrm{CN}$ ). Stars whose spectra are dominated by $\mathrm{TiO}$ and $\mathrm{VO}$ bands are oxygen-rich (i.e. they have a $\mathrm{C} / \mathrm{O}$ ratio $<1$ in their atmosphere), whereas stars whose spectra are dominated by features of $\mathrm{C}_{2}$ and $\mathrm{CN}$ are carbon-rich $(\mathrm{C} / \mathrm{O}>1)$. It turns out that all but one star in our sample displays $\mathrm{TiO}$ bands, this one star being BH Cru. This star has prominent $\mathrm{C}_{2}$ and (less prominent) $\mathrm{CN}$ features in its spectrum, whereas $\mathrm{ZrO}$ bands are absent. Additional very prominent features in $\mathrm{BH}$ Cru include the $\mathrm{Na} \mathrm{D}$ line, indicating a very low temperature and/or $\mathrm{C} / \mathrm{O}$ ratio, and the BaII 4554 and SrI 4607 lines, indicating an s-process enrichment. Figure 1 shows the Coralie spectrum of $\mathrm{BH} \mathrm{Cru}$, smoothed to the resolution of classification spectra to make the 
Table 2. Results of our analysis.

\begin{tabular}{|c|c|c|c|c|c|c|c|c|c|c|c|c|c|}
\hline Name & $\begin{array}{c}\text { Change } \\
\text { type }\end{array}$ & $\begin{array}{l}\text { Sp. } \\
\text { type }\end{array}$ & $\begin{array}{r}r v_{\text {helio }} \\
\left(\mathrm{km} \mathrm{s}^{-1}\right)\end{array}$ & Tc & Tc lit. & $\begin{array}{l}T_{\text {eff }} \\
(\mathrm{K})\end{array}$ & $\log \epsilon(\mathrm{Li})$ & $\begin{array}{c}\mathrm{d} \ln P / \mathrm{d} t \\
\left(10^{-3} \mathrm{yr}^{-1}\right)\end{array}$ & $\begin{array}{c}P \\
(\mathrm{~d}, \mathrm{~T} 05) \\
\end{array}$ & $\begin{array}{c}P \\
(\mathrm{~d})\end{array}$ & $J-K$ & $M_{\mathrm{K}}$ & $M_{\text {bol }}$ \\
\hline (1) & (2) & (3) & (4) & (5) & (6) & (7) & (8) & (9) & (10) & (11) & (12) & (13) & $(14)$ \\
\hline $\mathrm{BH} \mathrm{Cru}$ & $\mathrm{SCh}$ & $\mathrm{C}$ & -3.7 & yes & $\ldots$ & 3000 & -0.7 & +3.70 & 530 & 524.4 & 1.644 & -8.69 & -5.59 \\
\hline RU Vul & $\mathrm{SCh}$ & M2-4 & -67.9 & no & $\ldots$ & 3700 & $\lesssim-1.5$ & -7.11 & 112 & 108.8 & 1.223 & $-6^{\mathrm{m}} 88$ & -3.89 \\
\hline T UMi & $\mathrm{SCh}$ & M4-6 & +8.7 & no & $\ldots$ & 3300 & $\lesssim 0.0$ & -8.47 & 240 & 229.1 & 1.553 & -7.44 & $-4^{\mathrm{m}} \cdot 36$ \\
\hline R Aql & $\mathrm{CCh}$ & M5-9 & +59.3 & no & $\begin{array}{c}\text { prob }^{1} \\
\mathrm{dbffl}^{2} \\
\mathrm{no}^{3}\end{array}$ & 3000 & $\lesssim 0.0$ & -1.56 & 270 & 270.6 & 1.361 & $-7 \mathrm{~m} \cdot 68$ & $-4^{\mathrm{m}} \cdot 81$ \\
\hline R Hya & $\mathrm{CCh}$ & M6-9 & -15.9 & yes & $\begin{array}{c}\text { yes }^{4} \\
\text { prob }^{2} \\
\text { no }^{5}\end{array}$ & 3100 & $\lesssim 0.0$ & -0.71 & 385 & 376.6 & 1.261 & $-8 \mathrm{~m} \cdot 16$ & $-5^{\mathrm{m}} \cdot 17$ \\
\hline W Dra & $\mathrm{CCh}$ & M3-4 & -3.6 & $?$ & $\ldots$ & 2950 & $\lesssim 0.0$ & +1.03 & 282 & 289.6 & 1.340 & -7.78 & $-4^{\mathrm{m}} \cdot 74$ \\
\hline R Nor & $\mathrm{MCh}$ & M3-7 & -28.5 & no & $\ldots$ & 3200 & +4.6 & +0.29 & 510 & 496.2 & 1.310 & -8.55 & -5.50 \\
\hline S Her & $\mathrm{MCh}$ & M4-7.5S & -17.5 & yes & yes $^{2}$ & 3300 & $\lesssim 0.0$ & +0.06 & 307.5 & 304.1 & 1.166 & $-7^{m} \cdot 85$ & -4.89 \\
\hline S Ori & $\mathrm{MCh}$ & M5-10 & +34.5 & yes & $\mathrm{dbfl}^{2}$ & 2950 & +0.8 & +0.35 & 430 & 433.4 & 1.352 & -8.39 & $-5^{m} .49$ \\
\hline T Cep & $\mathrm{MCh}$ & M5-9 & -15.7 & yes & $\begin{array}{c}\text { prob }^{1} \\
\text { yes }^{2}\end{array}$ & 3000 & +0.5 & +0.12 & 390.9 & 386.6 & 1.328 & -8.19 & $-5 \cdot 16$ \\
\hline W Hya & $\mathrm{MCh}$ & M7-9 & +40.5 & no & $\begin{array}{c}\text { prob }^{1} \\
\text { no }^{6}\end{array}$ & 3300 & +0.5 & $\ldots$ & 361 & 388.6 & 1.459 & $-8 \mathrm{~m} 20$ & $-5 \cdot 25$ \\
\hline T Her & const. & M2-8 & -91.9 & no & $\mathrm{dbfl}^{2}$ & 3400 & +2.0 & +0.04 & 164.9 & 163.9 & 1.222 & $-6^{\mathrm{m}} \cdot 96$ & -4.20 \\
\hline
\end{tabular}

Notes. The meaning of the columns is as follows: (1) name listed in Simbad; (2) period change type; (3) range of spectral type; (4) heliocentric radial velocity; (5) presence of Tc, this paper; (6) presence of Tc, previous studies; (7) effective temperature; (8) abundance of Li; (9) rate of period change; (10) period as given in Templeton et al. (2005); (11) period found in this study; (12) mean $J-K$ colour; (13) absolute $K$ magnitude; (14) bolometric magnitude. References of previous studies on Tc content: ${ }^{(1)}$ Little-Marenin \& Little (1979); ${ }^{(2)}$ Little et al. (1987); (3) Vanture et al. (1991); ${ }^{(4)}$ Merrill (1952); ${ }^{(5)}$ Uttenthaler \& Lebzelter (2010); (6) Lebzelter \& Hron (1999). Abbreviations mean: "prob" = probably, "dbfl" = doubtful.

main features visible. The $\mathrm{C}_{2}$ bands clearly dominate the spectrum.

Among the other stars, the one with clearly the weakest $\mathrm{TiO}$ features is RU Vul, implying that it is the hottest star in our sample (see also Sect. 3.1.4). All other O-rich stars display pronounced bands of $\mathrm{TiO}$. The range of spectral types found in the compilation of Skiff (2010) is listed in Col. 3 of Table 2. All stars exhibit hydrogen emission lines from time to time.

\subsubsection{Technetium content}

Before searching for Tc, it is necessary to measure the radial velocity of the stars. To this end, we applied a cross-correlation technique, using a synthetic model spectrum as a template. The spectral range of $430-435 \mathrm{~nm}$ was used to complete this, except for W Dra, for which only the red part of the spectrum had enough signal and we then used the range $701-710 \mathrm{~nm}$. The found heliocentric radial velocities are summarised in Col. 4 of Table 2.

With the radial velocity information, we determined whether or not Tc is present in the sample stars using the flux-ratio method introduced by Uttenthaler et al. (2007a), and by visual inspection of the spectra around the strongest lines of neutral Tc $(\lambda=398.497,403.163,404.911,409.567,423.819,426.227$, and $429.706 \mathrm{~nm}$, in air). In this method, the mean flux contained within a range of a few wavelength pixels centred on a quasicontinuum point is divided by the mean flux in a small region around the Tc line. If Tc line absorption is present, the resulting ratio will be considerable larger than one, but close to one otherwise. The measured flux ratios for the two Tc lines at the longest wavelengths, hence highest $S N R$, are shown in Fig. 2. For the Tc $426.2 \mathrm{~nm}$ line, the range $426.120-426.140 \mathrm{~nm}$ was used for the continuum point and $426.220-426.240 \mathrm{~nm}$ for the line, while

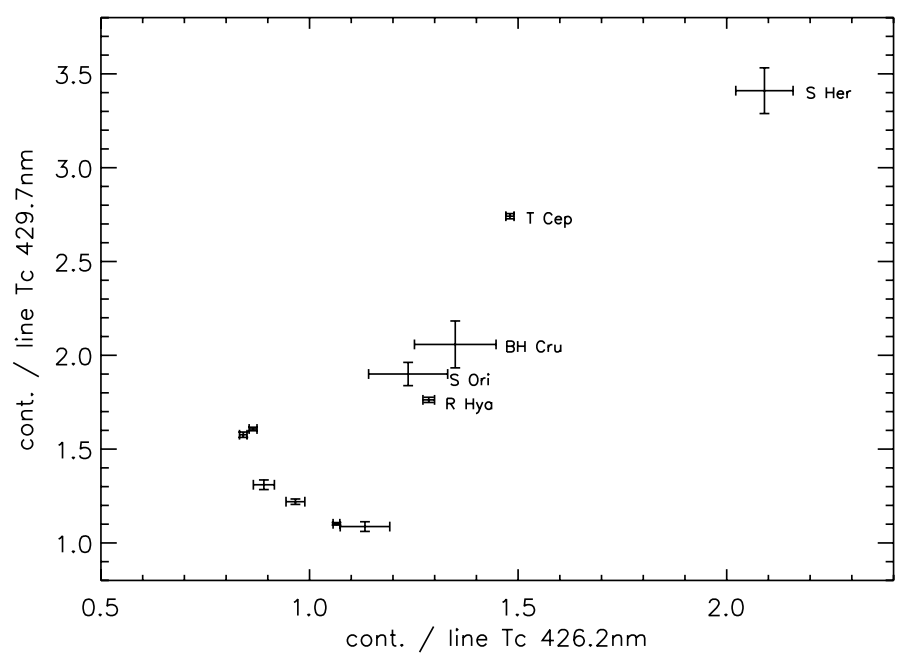

Fig. 2. Continuum-to-line flux ratios of the Tc lines at 426.2 and $429.7 \mathrm{~nm}$ of our sample stars. The data points of the Tc-rich stars, i.e. those stars with an enhanced ratio in both lines, are marked with their names.

for the Tc $429.7 \mathrm{~nm}$ line the ranges were $429.625-429.655$ and $429.690-429.718 \mathrm{~nm}$, respectively. The error bars of the data points were determined with a Monte Carlo simulation. Onehundred trial spectra were created by adding Gaussian noise with the magnitude of the inverse SNR to the observed, combined spectrum. The flux ratios were measured from those trial spectra, and the standard deviation of the distribution of the ratios was adopted as the error bar.

Five stars were found to have a higher continuum-to-line flux ratio, which was confirmed by visual inspection of the spectra 


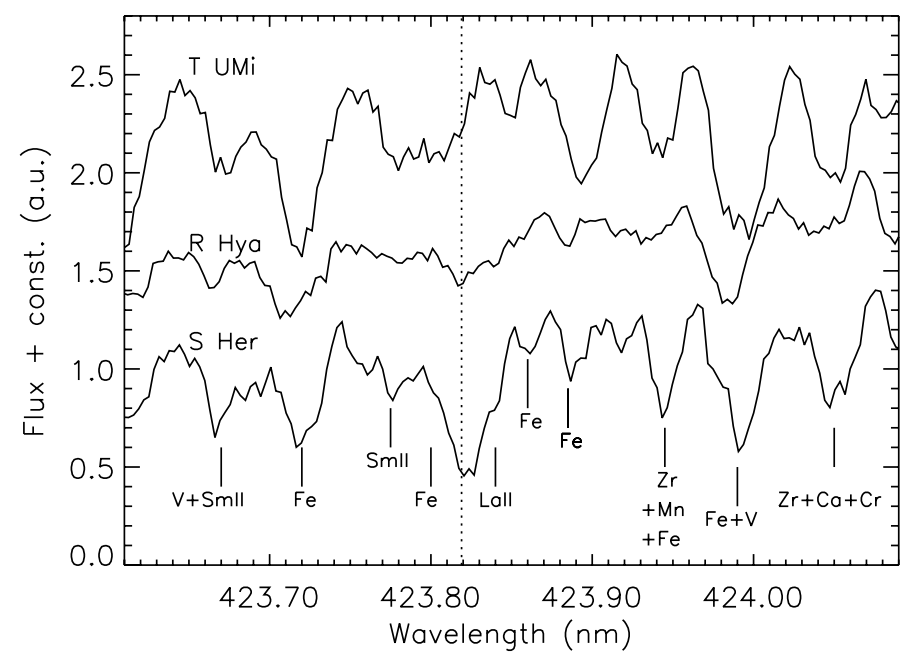

Fig. 3. Spectra of three sample stars around the Tc line at $423.819 \mathrm{~nm}$. From top to bottom: T UMi, R Hya, and S Her. The spectra of T UMi and R Hya have been shifted upwards by 1.2 and 0.6 to make the plot clearer. The wavelength of the Tc line is marked with a dotted vertical line.

around the Tc lines. These stars are BH Cru, R Hya, S Her, S Ori, and T Cep. The surprise in this list is R Hya, which was previously classified by Uttenthaler \& Lebzelter (2010) as Tc-poor on the basis of a UVES spectrum of that star. After careful inspection of all Tc lines in the observed range and a comparison between the UVES and the Hermes spectrum of R Hya, it became clear that this star does indeed have Tc in its atmosphere, in contrast to the findings of Uttenthaler \& Lebzelter (2010). The UVES observations on 6 July 2000 were performed near maximum light of R Hya, while the Hermes observations were done about half-way between a maximum and a minimum. A cross-correlation of the two spectra shows that there are velocity components in the atmosphere that differ by up to $12 \mathrm{~km} \mathrm{~s}^{-1}$. The lines in the Hermes spectrum also appear deeper, which is not simply a consequence of the somewhat lower resolution of the UVES spectrum (50000, compared to 85000 for the Hermes spectrum). Many lines in the UVES spectrum, including the Tc lines, seem to suffer from the often cited "line weakening" effect in Mira variables, which causes many spectral lines to become much weaker at times, or to disappear completely (Merrill et al. 1962). This seems to have happened with the near-maximum UVES spectrum of R Hya, which we have to re-classify as a Tc-rich star. This can also be seen as a warning that intrinsically weak lines (the Tc lines of R Hya are already at the weak end of the Tc line strengths observed in the current sample) may go entirely undetected if a Mira star is observed at a pulsation phase with severe line weakening. There was no error in the analysis of Uttenthaler \& Lebzelter (2010): revisiting the spectrum now, we would still classify it as Tc-poor. In view of this situation, we scrutinized the present sample to assess whether we could have mistaken a Tc-rich star for a Tc-poor one, but conclude that this is not the case.

In Fig. 3, the spectra of three sample stars around the Tc line at $423.819 \mathrm{~nm}$ are shown. The three stars are chosen to delineate a sequence of increasing Tc line strength from absent in T UMi, weak in R Hya, and strong in S Her. The lines in R Hya are generally weaker than in the other two stars, again illustrating the difficulty of detecting the Tc lines in this star. A few of the surrounding lines are identified, with identifications being taken from Davis (1947).

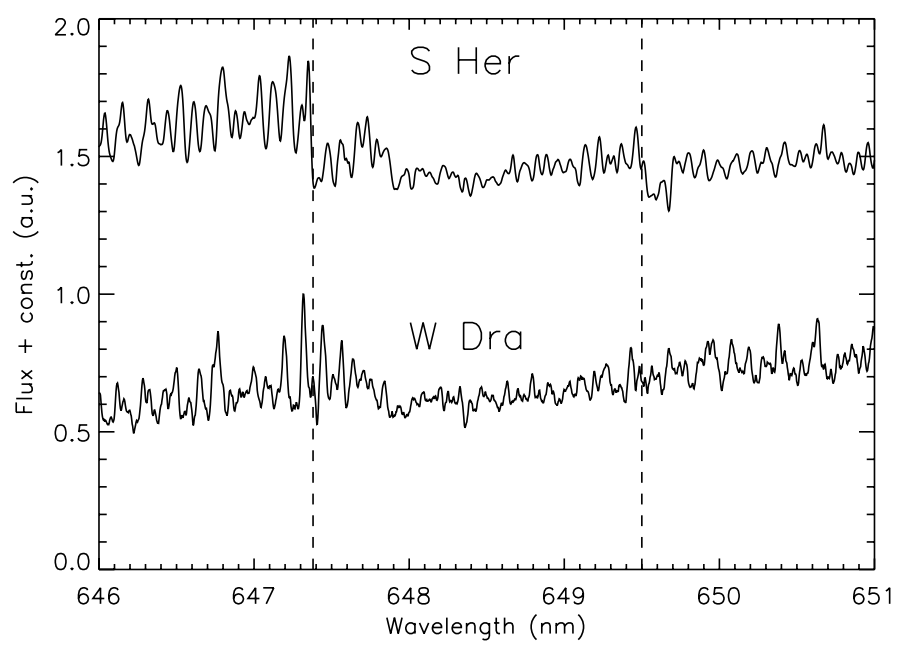

Fig. 4. Spectra of S Her (top) and W Dra (bottom) around the ZrO bandheads at 647.4 and $649.5 \mathrm{~nm}$, smoothed by a nine-point running boxcar. The theoretical wavelengths of the bandheads are marked by dashed lines. The feature at $647.9 \mathrm{~nm}$ is a $\mathrm{TiO}$ bandhead.

The results of our search for Tc are listed in Col. 5 of Table 2. Column 6 summarises what was previously reported in the literature about the Tc content of the sample stars. This shows that the Tc content of most stars in our sample was unclear, with there even being contradictory classifications. As discussed above, this reflects that the search for the weak Tc lines in crowded, low-SNR spectra of red giant stars is not a simple and straightforward task.

\subsubsection{Other s-process indicators}

The spectrum of W Dra has basically no signal at the wavelength of the Tc lines, hence we searched its spectrum for other indicators of $s$-process enrichment. The bands of the $\mathrm{ZrO}$ molecule are such an indicator, because $\mathrm{Zr}$ is also an $s$-process element. The formation of $\mathrm{ZrO}$ is also facilitated by an enhanced $\mathrm{C} / \mathrm{O}$ ratio, because at $\mathrm{C} / \mathrm{O} \lesssim 1$ the molecular equilibrium is shifted to $\mathrm{ZrO}$ and $\mathrm{LaO}$, at the expense of $\mathrm{TiO}$ and $\mathrm{VO}$. However, in contrast to $\mathrm{Tc}, \mathrm{Zr}$ is a stable element, hence is not an indicator of a recent $s$-process and 3DUP. An enhanced $\mathrm{Zr}$ abundance can also be caused e.g. by the mass transfer of $s$-process enriched material from a binary companion, which would now be a white dwarf. The mass transfer could have happened billions of years ago. While Tc would have decayed by now, $\mathrm{Zr}$ would still be present in the atmosphere of the now evolved secondary star. This binary mass transfer scenario is the probable mechanism leading to Tc-poor S-type stars (Van Eck \& Jorissen 1999). The absence of $\mathrm{Zr}$ enrichment (and hence of $\mathrm{ZrO}$ bands) would however indicate that neither intrinsic nor extrinsic $s$-process enrichment has taken place in a given star.

Particularly strong and weakly blended bandheads of $\mathrm{ZrO}$ are to be found in cool star spectra at wavelengths of 647.4 and $649.5 \mathrm{~nm}$. The combined spectrum of W Dra has enough flux at this wavelength to detect the $\mathrm{ZrO}$ bands if they were present, but apparently they are not (Fig. 4). In addition, the spectra of all other sample stars were inspected for the $\mathrm{ZrO}$ bands, but only $\mathrm{S}$ Her definitely shows them ${ }^{1}$. As seen from Fig. 2, S Her is also the star with the strongest Tc lines in our sample, so it is clearly of MS spectral type. Since $\mathrm{ZrO}$ is not detectable in three of the

1 As discussed in Sect. 3.1.1, BH Cru is C-rich, which leaves no trace of O-bearing molecules such as $\mathrm{ZrO}$. 
Tc- and O-rich sample stars, the absence of $\mathrm{ZrO}$ bands does not imply the absence of Tc. With $\mathrm{ZrO}$ being absent in W Dra, we cannot draw a firm conclusion about the Tc content of this star. We can only conclude that W Dra is an O-rich, M-type star, without strong $s$-process (and $\mathrm{C} / \mathrm{O}$ ) enhancement.

\subsubsection{Lithium abundance}

In contrast to $\mathrm{Tc}$, we can determine for Li not only its presence but also its abundance, because state-of-the-art model spectra of cool giants are able to reproduce observed spectra much more closely in the region of the Li line than around the Tc lines. The most important stellar parameter that determines the strength of the Li I resonance doublet at $670.8 \mathrm{~nm}$ is the effective temperature. For the O-rich stars in our sample, the temperature can be determined from the strength of the TiO bands. Our method for temperature determination involves a grid of COMARCS model atmospheres and spectra (Aringer et al. 2009), and closely follows the approach of García-Hernández et al. (2007). Details of the method can be found in Uttenthaler \& Lebzelter (2010), and here we only outline the main steps of the procedure.

A grid of synthetic spectra based on model atmospheres with temperatures between 2600 and $3600 \mathrm{~K}$ was calculated. The step width in temperature was $100 \mathrm{~K}$, and two models were added at 2950 and $3150 \mathrm{~K}$. A number of other parameters, which have a much smaller impact on the spectrum than the temperature, have been fixed beforehand to realistic values. The adopted parameter values are: $M=1 M_{\odot}, \log g\left[\mathrm{~cm} \mathrm{~s}^{-2}\right]=0.0, Z=Z_{\odot}=0.0156$, $\mathrm{C} / \mathrm{O}=0.48\left(\operatorname{solar}^{2}\right)$, and micro-turbulence $\xi=2.5 \mathrm{~km} \mathrm{~s}^{-1}$. The spectra were calculated for regions that contain band heads of the TiO molecule, namely 668.4-674.0 (which also includes the Li line itself) and 701-710 nm. Before fitting the synthetic spectra to the observed ones, they were smoothed with a Gaussian kernel to the resolution of the respective instrument. An additional macro-turbulence had to be adopted, with the maximum value of $9 \mathrm{~km} \mathrm{~s}^{-1}$ for $\mathrm{R}$ Nor. The synthetic spectra were then fit to the observed spectra using a $\chi^{2}$ minimisation method. The temperature of the model spectrum that most closely reproduced a given observed spectrum was adopted as the effective temperature of that star. These temperatures are listed in Col. 7 of Table 2.

Three exceptions to this general procedure have to be noted. The Coralie spectrum of R Nor does not reach beyond $690 \mathrm{~nm}$, thus the temperature was determined only from the region 668.4-674.0 $\mathrm{nm}$. RU Vul has too weak TiO bands to reliably determine the temperature from them. From its mean spectral type (M3) and the calibration in Fluks et al. (1994), a temperature of $3666 \mathrm{~K}$ can be inferred. We adopted $3700 \mathrm{~K}$ for the spectral synthesis. At this temperature, the $\mathrm{TiO}$ bands predicted by the synthetic spectra (which are based on solar metallicity) are much too strong. However, the temperature may also not be much higher than this because the TiO bands, the classification criterion of $\mathrm{M}$ stars, are clearly present in RU Vul, albeit weak. The observed spectrum in the two spectral pieces can only be fit reasonably well if we assume a metallicity $1.5 \mathrm{dex}$ below the solar value. A comparison with the hotter, metal-poor giant Arcturus $\left(T_{\text {eff }}=4290 \mathrm{~K},[\mathrm{M} / \mathrm{H}]=-0.5\right)$ also shows that RU Vul has much weaker metal lines. Although we did not make a full metallicity determination, we suggest that RU Vul is a quite metal-poor star. Except for RU Vul, the temperature range of

\footnotetext{
2 A C/O ratio fixed to the solar value can be problematic for MSor S-stars. However, we have only one MS-star in our sample (S Her, which shows $\mathrm{ZrO}$ bands), and it turns out that its $\mathrm{Li}$ line is undetectable, independent of the assumed $\mathrm{C} / \mathrm{O}$ ratio.
}

our sample stars is fairly narrow. The C-star BH Cru does not exhibit the temperature-sensitive $\mathrm{TiO}$ bands, so we adopted the temperature of $3000 \mathrm{~K}$ reported by Zijlstra et al. (2004). For the spectral synthesis, we used model no. 443 from Aringer et al. (2009), which has $T_{\text {eff }}=3000 \mathrm{~K}$, a C/O ratio of 1.05 , and otherwise identical parameters to our grid models. A C/O ratio higher than 1.05 does not significantly change the spectral appearance in the vicinity of the $\mathrm{Li}$ line, hence has very little influence on the measured Li abundance.

In the next step, spectra with varying amounts of Li were synthesised based on the best-fit model atmosphere. The hyperfine structure of the Li line was taken into account in the spectral synthesis, but not the isotopic shift (i.e. only the contribution by ${ }^{7} \mathrm{Li}$ was included). This effect can be neglected here, because the ${ }^{6} \mathrm{Li}$ abundance can be expected to be very small, and other effects such as the (velocity) structure of the atmosphere, blending with $\mathrm{TiO}$ lines, etc., play a much more important role. The Li abundance that yielded a minimum in the residuals of observed calculated flux was adopted as the measured Li abundance and is reported in Col. 8 of Table 2. The uncertainty in the Li abundance is however large, up to 0.6 dex (García-Hernández et al. 2007; Uttenthaler \& Lebzelter 2010). The largest part of this uncertainty is based on the uncertainty in the temperature $(0.4 \mathrm{dex})$, the other stellar parameters and flaws in the (TiO) line lists contribute to the rest.

Because of the large opacity of the TiO molecule relative to that of $\mathrm{C}_{2}$ and $\mathrm{CN}$ at the wavelength of the Li doublet (a factor of $\sim 10$ difference at $T_{\text {eff }}=3000 \mathrm{~K}$ !), the Li detection threshold for O-rich stars is much higher than for C-rich stars. Hence, the abundance of $\log \epsilon(\mathrm{Li})=-0.7$ measured in BH Cru would not be detectable in M-type stars of similar temperature. Kipper \& Wallerstein (1990) measure a $\mathrm{Li}$ abundance in BH Cru of -1.5 dex relative to the Sun, or -0.4 on the $\log \epsilon$ scale, in fairly good agreement with our result. The good agreement is remarkable because $\mathrm{BH}$ Cru has changed its spectral type considerably over the past few decades, and our spectrum was taken almost 26 years after the one used by Kipper \& Wallerstein (1990). Furthermore, the synthetic spectrum based on the model chosen for $\mathrm{BH} \mathrm{Cru}(\mathrm{C} / \mathrm{O}=1.05)$ closely reproduces the molecular lines $\left(\mathrm{C}_{2}, \mathrm{CN}\right)$ in the observed spectrum in the vicinity of the $\mathrm{Li}$ line, which is additional support for a $\mathrm{C} / \mathrm{O}$ ratio $>1$ in that star.

Irrespective of the metallicity of RU Vul, the Li line in this star is undetectable. Because of its higher temperature and less crowded spectrum, an upper limit of $\log \epsilon(\mathrm{Li}) \lesssim-1.5$ appears reasonable.

The only period change class with noteworthy amounts of $\mathrm{Li}$ are the meandering Miras. In this group, only S Her does not appear to have $\mathrm{Li}$, whereas $\mathrm{R}$ Nor has a huge amount of it. The measured abundance is $\log \epsilon(\mathrm{Li})=+4.6$, which is higher than the solar photospheric abundance by a factor of $\sim 3200$ ! The observed spectrum of $\mathrm{R}$ Nor together with a synthetic spectrum to fit the Li line is shown in Fig. 5. With the adopted abundance, only the red wing of the line is reproduced well, whereas the blue wing would require an even higher abundance. However, some of the absorption in the blue wing probably stems from circumstellar absorption in the expanding stellar wind. This is supported by the profile of the NaI D doublet, which also exhibits a strong blue-shifted absorption component. We did not attempt to fit the blue-shifted component in the Li line, because our model atmospheres do not include circumstellar shells. The very high $\mathrm{Li}$ abundance in $\mathrm{R}$ Nor suggests that it is undergoing $\mathrm{HBB}$, and that it is a fairly massive star. R Nor is in some respects similar to the very Li-rich S-star V441 Cyg, but is not $s$-process enriched (Uttenthaler \& Lebzelter 2010). 


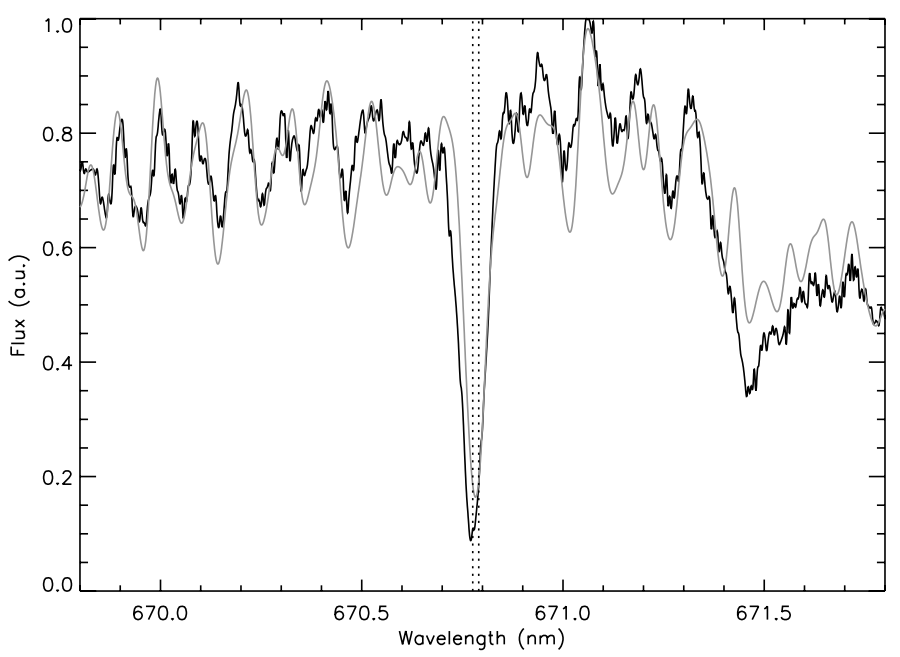

Fig. 5. Observed spectrum of R Nor around the Li line doublet (black graph), together with a synthetic spectrum (grey graph). The synthetic spectrum is based on a COMARCS model atmosphere with $T_{\text {eff }}=$ $3200 \mathrm{~K}, \log g=0.0$, solar metallicity, and solar C/O ratio. A Li abundance of $\log \epsilon(\mathrm{Li})=+4.6$ was assumed in the spectral synthesis. The dotted vertical lines indicate the laboratory wavelengths of the Li line doublet. All other features in the plotted range are due to TiO.

Interestingly, the Tc-poor, constant-period Mira T Her also contains a considerable amount of $\mathrm{Li}$. It is thus similar to the Galactic bulge AGB star Plaut 3-45 discussed in Uttenthaler et al. (2007b). This star could be another example of a low-mass AGB star experiencing extra-mixing, thereby producing $\mathrm{Li}$.

\subsection{Present-day periods}

Most of our sample stars have experienced a considerable change in pulsation period in the past few decades and centuries. To check whether or not this period change has continued in recent years, we analysed the AAVSO visual light curves to determine the present-day periods. We downloaded from the AAVSO web page ${ }^{3}$ the visual and $V$-band observations between JD 2452000 and JD 2455500 , i.e. between 31 March 2001 and 31 October 2010, and completed a Fourier analysis on the unprocessed data with the program period04 (Lenz \& Breger 2005). The detected periods (in days) are summarised in Col. 11 of Table 2. The peaks in the Fourier spectrum are fairly sharp, the Monte Carlo simulation performed with period04 yielded period uncertainties of or less than 1 day. Only the Fourier spectrum of RU Vul has no clearly highest peak. The one at $108.8 \mathrm{~d}$ is only barely higher than other peaks, and is broad and asymmetric. We thus conclude that no clear periodic pulsation is present any more in this star.

Templeton et al. (2005), who use AAVSO data up to about 11 September 2003, do not list present-day periods, but instead list average periods over the entire AAVSO time series available for a particular star (which can be between a few decades and a century long). Nevertheless, they do provide diagrams of the period evolution for the stars with the largest period change, which are useful to check whether the period change has continued. The last period value plotted in these diagrams is the one listed in Col. 10 of Table 2. The meandering Miras S Her and T Cep and the "constant" Mira T Her were not found to have a large period change (on average over the total time span of AAVSO data)

\footnotetext{
${ }^{3}$ http://www . aavso.org
}

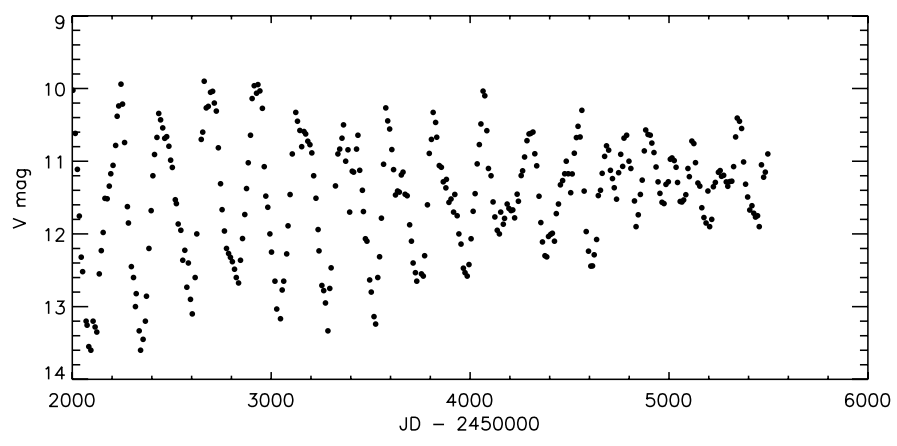

Fig. 6. AAVSO light curve of T UMi since JD 2452000 . The data points are 10 -day means. The previously dominant period of $\approx 230 \mathrm{~d}$ has disappeared in the most recent data.

by Templeton et al. (2005), thus no such plots are available for them, and we instead list the average period given in Templeton et al. (2005). W Hya was not included in the survey of Templeton et al. (2005), probably because it is classified as SRV in Simbad. This classification is controversial, and we discuss it further in Sect. 4.1. In any case, we decided to list in Table 2 the period of W Hya as given in the General Catalogue of Variable Stars (Samus et al. 2009). In this table (Col. 9), we also indicate the rate of period change, $\mathrm{d} \ln P / \mathrm{d} t$, defined as the slope of the period divided by the average period (Templeton et al. 2005). For RU Vul, this rate is taken from Templeton et al. (2008), and for W Hya the rate is missing.

From these data, we can make the following observations. Among the SCh change type stars, BH Cru seems to have stopped or maybe even reversed its period increase. A deceleration of the period increase was proposed by Templeton et al. (2005). RU Vul has continued its period decrease (also found by Templeton et al. 2008), and the pulsation is very weak now. It is not detectable any more by visual observations in the most recent data. The most dramatic evolution in recent years has however been seen in T UMi. In the time span investigated here, its dominant period decreased to $229 \mathrm{~d}$ and did not stop there. In the most recent data we see that the star might even have switched its pulsation mode from Mira-like to semi-regular variability around JD 2454600 (May 2008), as the dominant period made a jump to $113.6 \mathrm{~d}$ ! A period ratio close to 2 can be expected for a switch from fundamental mode (Mira-like) to first overtone (SRV) pulsation (Wood et al. 1991). At the same time, the full amplitude decreased to about $1 \mathrm{~m} .5$. According to the classical (but arbitrary) definition of $\Delta V>2$. 5 , this small amplitude does not qualify T UMi as a Mira any more, but as SRV. The light curve of T UMi in the time span investigated here is displayed in Fig. 6. Continued photometric monitoring will be very important to follow the exciting evolution of this star.

Among the CCh Miras, R Hya has continued to decrease its period, and W Dra has continued to increase it, while R Aql's period remained practically constant. This is somewhat in contradiction to the luminosity evolution predicted for these stars by Wood \& Zarro (1981), as the period of W Dra is expected to stop increasing at some point, whereas R Hya and R Aql are expected to continue their decrease, albeit at a somewhat slower rate. The development on these short timescales may however not be regarded as a stringent constraint of the TP model employed by Wood \& Zarro (1981). Among the MCh Miras, R Nor and $\mathrm{W}$ Hya are the stars that display the strongest changes. The former decreased its period in the recent years, while the latter increased it considerably. 


\subsection{Luminosities}

To compare the evolutionary states in the HRD of the different period change classes, as well as of the Tc-rich and the Tc-poor stars, we determined the absolute bolometric magnitudes (luminosities) of our sample stars. In principle, this could be done very easily using a period-luminosity relation for Mira stars, e.g. the one from Guandalini \& Busso (2008, their Fig. A.1). However, our sample stars do not necessarily closely follow this relation, because of the change in the pulsation period. For example, Zijlstra et al. (2004) argue that the near-IR data of BH Cru is consistent with evolution at constant luminosity, and that any increase in luminosity did not exceed 10 percent. If this were true, then the star would now be fainter than other stars of a similar pulsation period that do follow the Mira period-luminosity relation. The only way to determine luminosities independently of the pulsation period is by the measurement of the parallax of the stars. Unfortunately, parallaxes of high significance $\left(\sigma_{\pi} / \pi<0.5\right)$ are available for only four of our sample stars (Pourbaix et al. 2003).

In view of this situation, we decided anyway to infer the luminosity from the pulsation period. In a first step, we used the $\log P-K$ relations of Riebel et al. (2010) to determine the absolute $K$ magnitudes. As these relations are based on LMC stars, we assume that the same relation is applicable to Galactic AGB stars. This will likely introduce only a small uncertainty in the final luminosity. For the carbon star BH Cru, the relation for sequence 1 (classical Mira sequence) of C-rich stars, and for all other stars sequence 1 of the O-rich stars was used. As the period of RU Vul is no longer clearly detected in the most recent photometric data, we adopted the period of $155 \mathrm{~d}$ that it had before the period decline (Templeton et al. 2008) and forced it to follow also sequence 1 of the fundamental mode pulsators. This choice is justified in Sect. 4.1. A distance modulus to the LMC of 18.5 was assumed. To get from absolute $K$-magnitudes to the absolute bolometric magnitude $M_{\mathrm{bol}}$, we applied the $\mathrm{BC}(\mathrm{K})$ relations of Kerschbaum et al. (2010) based on the $J-K$ colour. Quasi-simultaneous photometry in the $J$ and $K$ bands was collected from the works of Catchpole et al. (1979), Fouqué et al. (1992), Kerschbaum \& Hron (1994), Whitelock et al. (2006), and the 2MASS catalogue (Skrutskie et al. 2006), transformed to the 2MASS photometric system with the relations given by Carpenter (2001), and averaged to a final value. For some stars, only the 2MASS data were available, but for most stars this resulted in a cycle-averaged $J-K$ colour. As in Kerschbaum et al. (2010), no correction for interstellar reddening was applied, because $E(J-K)$ is only a few 0 . 01 for these solar neighbourhood stars and variability and individual circumstellar extinction have a much larger influence. The $\mathrm{BC}(\mathrm{K})$ correction applied ranged between 2.76 and 3.11 . The mean $J-K$ colour, the absolute $K$ magnitude, and the resulting bolometric magnitude $M_{\text {bol }}$ are reported in Cols. 12-14 of Table 2. The $M_{\text {bol }}$ derived in this way differ on average by less than 0.08 from what is found by using the relation of Guandalini \& Busso (2008). We estimate a typical uncertainty of $\sim 0$. 16 in the bolometric magnitude from combining the uncertainties in the $\log P-K$ and $\mathrm{BC}(\mathrm{K})$ relations. The variable period of the stars might introduce an additional uncertainty, which is hard to quantify.

For the four stars in our sample with a good parallax (R Aql, $\mathrm{R}$ Hya, T Cep, and W Hya), we can compare the absolute $K$-magnitude derived from the $\log P-K$ relation with that derived from the parallax. Only in the case of W Hya do the values not agree within the error bars, $M_{K}$ from the $\log P-K$ relation being brighter by 0.36 than the brightest $M_{K}$ allowed by

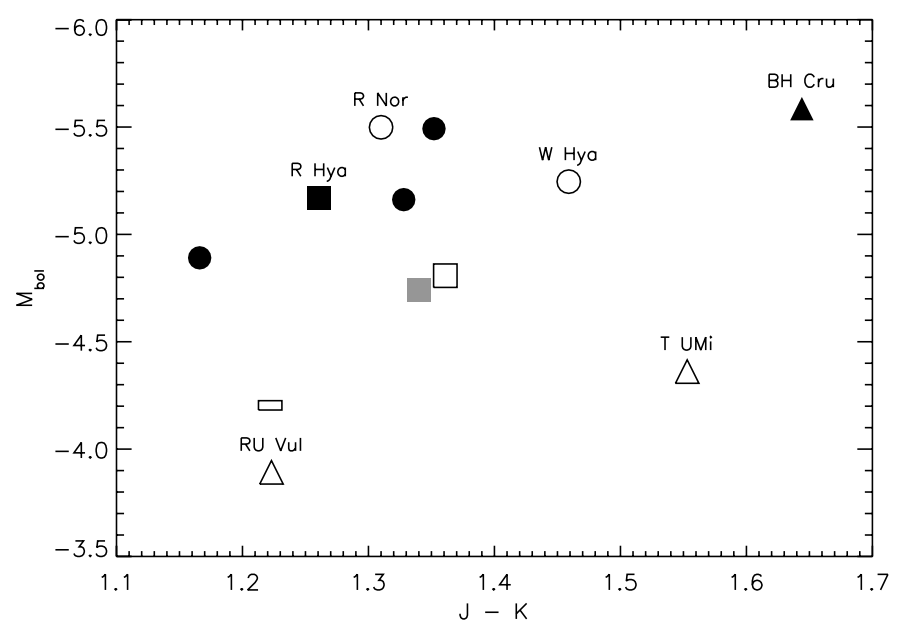

Fig. 7. HRD with our sample stars. The symbols are as follows: Triangles represent the SCh stars, squares the CCh Miras, circles the MCh Miras, and the rectangle represents the constant Mira T Her. Open symbols are the Tc-poor stars, filled symbols Tc-rich. The gray square is W Dra, for which no definite conclusion on its Tc content could be drawn. Stars discussed individually in Sect. 4.1 are identified with their names.

the $1 \sigma$ uncertainty in the parallax. The average difference for the other three stars is 0.18 , in good agreement with the error estimated for the luminosities adopted here. For R Nor, the uncertainty in the parallax is slightly larger than half the parallax itself. Nevertheless, a comparison of the $M_{K}$ magnitudes derived with the two methods is interesting and suggests that the true parallax is probably near the lower limit of the $1 \sigma$ range, hence the distance is probably near the upper limit of $800 \mathrm{pc}$ (see also Sect. 4.1).

The resulting HRD of our sample stars, using $J-K$ as a proxy for the temperature, is shown in Fig. 7. As already noted by Uttenthaler et al. (2007a), the Tc-rich stars seem to form the upper envelope of the distribution. They are the brightest stars at any given $J-K$ colour (or temperature). The only exception here is R Nor, a very bright but definitely Tc-poor star. This is further independent evidence that $\mathrm{R}$ Nor is a massive star with HBB (see Sect. 4.1). From its location in the HRD, W Dra falls among the Tc-poor stars, which suggests that it is also Tc-poor.

\section{Discussion}

Before we discuss the evolutionary states of the different period change classes, we first comment on the evolutionary states of a few remarkable individual objects in the sample.

\subsection{The evolutionary states of individual objects}

BH Cru displays Tc in its spectrum, a clear sign that it is on the TP-AGB. An $s$-process enhancement in that star has already been reported by Abia \& Wallerstein (1998). The pulsation period of BH Cru has increased considerably since 1970, but has been stable for some 15 years, or even slightly decreased. We presented in Sects. 3.1.1 and 3.1.4 evidence that BH Cru is a carbon star. It was initially classified as being of SC spectral type (with C/O essentially unity; Catchpole \& Feast 1971; Keenan \& Boeshaar 1980), but its type changed to CS and it experienced a marked period increase (Lloyd Evans 1985). Zijlstra et al. (2004) interpret this spectral change as being due to a 
decrease in the effective temperature by some $200 \mathrm{~K}$, rather than a change in $\mathrm{C} / \mathrm{O}$ ratio (i.e. by a recent dredge-up of $\mathrm{C}$ ). The same authors obtained a low-resolution spectrum of $\mathrm{BH}$ Cru in the range 600-700 $\mathrm{nm}$ and noted that $\mathrm{C}_{2}$ bands are not clearly present. However, they also stated that Loidl et al. (2001) found that $\mathrm{C}_{2}$ bands are present longward of $700 \mathrm{~nm}$. We compared the spectrum of BH Cru to Hermes spectra of well-known carbon stars and found that RS Cyg, a carbon star of spectral type C8.2, has an almost identical spectrum. The measured $\mathrm{C} / \mathrm{O}$ ratios of C-stars are clearly in excess of 1 (e.g. Lambert et al. 1986; Lederer et al. 2009). We favour the interpretation that the change in spectral type observed in the past few decades is really due to a recent 3DUP event, and not simply a temperature decrease. If that were the case, then every normal carbon star could become either a CS or an SC star simply by increasing its effective temperature. Dedicated modelling of the atmospheres and spectra of cool giant (Mira) stars with $\mathrm{C} / \mathrm{O}$ ratio close to unity, including non-equilibrium chemistry, is required to gain more certainty in this respect. We note that an increase in the $\mathrm{C} / \mathrm{O}$ ratio from below to above unity at constant luminosity naturally leads to an increase in the pulsation period, because of the associated change in chemistry and opacities. This is suggested by the linear pulsation models employed by Lebzelter \& Wood (2007), who take into account the effect of the $\mathrm{C} / \mathrm{O}$ ratio on the opacities and the atmospheric structure of pulsating AGB stars. This effect remains to be confirmed with more realistic non-linear pulsation models. If confirmed, this would mean that the observed period change in BH Cru can actually be caused by the dredge-up of carbon and the thereby changing opacities, rather than by the associated TP itself.

RU Vul no longer displays any clearly detectable pulsation, and shows signs of neither Tc nor Li. We find that it has a very low metallicity of $[\mathrm{M} / \mathrm{H}] \approx-1.5$. This agrees with the finding of Mennessier et al. (2001), who assign it to the extended (thick) disk, which is an old, metal-poor population. The same authors determine an absolute IRAS 12 mag of RU Vul (based on the statistical properties of the extended disk sub-sample), from which we infer a distance of $2070 \mathrm{pc}$ to that star. If we assume a fundamental mode pulsation with a period of $155 \mathrm{~d}$ (Templeton et al. 2008), use relation 1 of Riebel et al. (2010) to derive an absolute $K$-magnitude, and combine this value with the observed 2MASS $K$-magnitude, we arrive at exactly the same distance. This provides good confidence that assigning RU Vul to the fundamental mode pulsators is the correct choice. Kerschbaum \& Hron (1992) find that the SRa's are not a distinct class of variables but a mixture of "intrinsic" Miras and SRb's. Before its period and amplitude decline, RU Vul exhibited a quite regular light change with a visual amplitude of about $2 \mathrm{~m} 0$, somewhat below the (arbitrary) definition limit of Miras, and was classified as SRa. Furthermore, many short-period, small-amplitude Miras are known to belong to an old, metal-poor population (Hron 1991). We conclude that RU Vul is also representative of this very same population, but it failed to be classified as Mira because of its too low pulsation amplitude. Its supposedly high age also suggests that RU Vul is of relatively low mass $\left(\sim 1 M_{\odot}\right)$.

T UMi is an oxygen-rich, M-type star without any signs of $s$-process enrichment. It has thus not undergone a 3DUP event. The change in the pulsation behaviour of this star is dramatic. Before 1980, the period was fairly stable at $\sim 315 \mathrm{~d}$, when it started to decline at a high rate. By mid 2008, its period had decreased to $\sim 230 \mathrm{~d}$, when it seems to have switched the pulsation mode from Mira-like to semi-regular variability (Fig. 6). The present-day period is around $113 \mathrm{~d}$, with a visual amplitude below the definition limit of Miras. Whitelock (1999) already speculate that T UMi could drop out of the Mira instability strip in the HRD, which we confirm herewith. Mattei \& Foster (1995) suggest that it is in the early helium-shell flash phase where the period is expected to change most rapidly as the luminosity drops. An indication for a luminosity drop in T UMi was found in the detailed light curve analysis by Szatmáry et al. (2003), who also suggest that the period decrease should stop in the near future, which is however not (yet) the case. However, from the lack of Tc (and also Li) it seems that T UMi is not as highly evolved as other stars in the present sample, or that it is not massive enough to undergo a 3DUP event. If a recent TP is the cause of its period change, it is possibly an early (weak) TP.

$\mathrm{R}$ Hya is the longest-known Mira to have a steadily decreasing pulsation period. At the time of its discovery around AD 1700, it had a period of $\sim 500 \mathrm{~d}$, which then decreased to its present value of $\sim 376 \mathrm{~d}$, and might well continue to decline in the future. We find Tc at a low level in this star, which confirms its evolutionary state on the TP-AGB. We thus conclude that the TP scenario for the period change in this star is likely.

The high $\mathrm{Li}$ abundance in $\mathrm{R}$ Nor (Sect. 3.1.4) could be a result of hot bottom burning (HBB) going on in this star. This would require the star to have a mass of $\sim 4 M_{\odot}$. Lebzelter et al. (2005) suggest that R Nor is relatively massive (3-5 $M_{\odot}$ ), because it has a secondary maximum in its light curve, which is also found in luminous LMC Miras (see also McSaveney et al. 2007). This would be in line with our finding. In the LMC, the very Li-rich AGB stars are mainly found in the luminosity range $-6.0 \gtrsim M_{\text {bol }} \gtrsim-7.2$ (Smith et al. 1995). R Nor is somewhat below this limit $\left(M_{\text {bol }}=-5\right.$. 50, Sect. 4.1$)$, with the uncertainty that we have to rely on the period to determine its luminosity. Another indicator of a high mass can be a relatively small distance from the Galactic plane. The Galactic latitude of R Nor is $5^{\circ}$ and its parallax $\pi=2.76 \pm 1.71$ mas (Pourbaix et al. 2003). Even though the parallax is very uncertain, this would place $\mathrm{R}$ Nor at a distance from the Galactic plane between 20 and $70 \mathrm{pc}$ (where the larger distance is the more likely one, see Sect. 3.3). For Miras with periods in the range 300-400d, the exponential scale height is close to 240 pc (Jura \& Kleinmann 1992), hence $\mathrm{R}$ Nor is relatively close to the Galactic plane. Furthermore, García-Hernández et al. (2007) find no $s$-process enrichment in their sample of Li-rich, massive Galactic AGB stars. This could be explained by the presence of a massive convective envelope, which would strongly dilute any material dredged-up from regions close to the core. In addition, 3DUP could be very inefficient in high mass stars. An additional piece of evidence of a high mass would be an enhanced rubidium $(\mathrm{Rb})$ abundance, which is synthesised by the ${ }^{22} \mathrm{Ne}(\alpha, n)^{25} \mathrm{Mg}$ neutron source reaction (García-Hernández et al. 2006). Unfortunately, our Coralie spectrum of $\mathrm{R}$ Nor does not cover the $\mathrm{Rb}$ line at $780 \mathrm{~nm}$, so we cannot measure its abundance in this star. Nevertheless, there is compelling evidence that $\mathrm{R}$ Nor is of high mass, probably around $4 M_{\odot}$.

Whether W Hya is an SRV or a Mira is not entirely clear. This question was discussed in detail by Nowotny et al. (2010). From its light variation and location in the P-L diagram, W Hya would indeed be classified as a Mira. From the velocity variations in its atmosphere, on the other hand, it can clearly be assigned to the group of SRVs. Lebzelter et al. (2005) conclude that a high mass might be the reason for the remarkable velocity behaviour. Although some Li is present in the atmosphere of W Hya, the mass of this star is probably too low for efficient operation of $\mathrm{HBB}$. 


\subsection{The evolutionary states of the period change classes}

The SCh group in our sample is very heterogeneous: The period of the C- and Tc-rich Mira BH Cru is (or has been) increasing, the O-rich, Tc-poor stars T UMi and RU Vul have decreased their periods considerably. In addition, the derived luminosities (Sect. 3.3) differ widely. These three stars differ quite fundamentally from each other, including in terms of their evolutionary states. Nevertheless, they show similarly large period change rates (in absolute terms). If their period change is caused by a $\mathrm{TP}$, the inhomogeneity of the presence of Tc in the SCh group suggests that a TP with an associated large period change can happen before a star undergoes a 3DUP event.

From the viewpoint of chemistry and luminosity, the CCh and the MCh groups are much more homogeneous than the $\mathrm{SCh}$ group. All members of these two groups studied here are late M-type stars. However, both the $\mathrm{CCh}$ and the $\mathrm{MCh}$ groups are inhomogeneous in terms of the presence of Tc, even though for W Dra no definite conclusion could be drawn. There is some evidence that the MCh group of stars is on average more massive than the other two groups. The periods in this group are fairly long, between 300 and $500 \mathrm{~d}$, although this is in some contradiction to Zijlstra \& Bedding (2002), who note that all meandering Miras have periods of $400 \mathrm{~d}$ or longer. Four of five stars in this group have at least some $\mathrm{Li}$, and three display Tc. The presence of Tc certifies that they are definitely in the TPAGB phase. We do, however, not suggest that the period change in the MCh group is connected to a recent TP, as their periods change back and forth on a much too short timescale. One of the alternative explanations presented in the introduction (feedback between pulsation and atmospheric structure or chaotic behaviour) might hold for them.

\section{Summary and conclusions}

From our search for Tc and other $s$-process indicators, as well as for $\mathrm{Li}$, in the spectra of Mira variables with changing pulsation periods, we can draw several conclusions. In our sample of twelve stars, have identified five that display Tc absorption lines, indicating that they have undergone a 3DUP event following a TP. The most important conclusion, however, is that there is no clear correlation between the presence of Tc and the type of period change, even in this limited sample. We suggest that the two Tc-rich, non-meandering Miras BH Cru and R Hya are the most likely to have experiences a recent TP, although their period change rates are quite different. Furthermore, we presented evidence that $\mathrm{BH}$ Cru changed its spectral type from SC to $\mathrm{C}$, which indicates that there has been a recent 3DUP that raised its $\mathrm{C} / \mathrm{O}$ ratio above unity. There is also accumulating evidence (high Li abundance, high luminosity, pulsation behaviour, proximity to the Galactic plane) that R Nor is a fairly massive star of $\sim 4 M_{\odot}$. On the basis of an analysis of AAVSO data of T UMi, we report that this star probably switched its pulsation mode from Miralike to SRV very recently. Finally, we suggest that RU Vul is a representative of the old, metal-poor thick disk of a consequently low mass.

Continuous photometric monitoring of Mira variables, in particularly those with large period changes, is mandatory to gain further insight into the AGB phase of stellar evolution. The archives of amateur observations such as those at AAVSO are an invaluable resource of data for this study. In parallel to photometric monitoring, we also recommend spectroscopic monitoring of the stars with changing pulsation period, albeit with sparser sampling of a few years. This will be of high importance to check whether the period change is also accompanied by a spectral change.

In addition to the prominent and well-studied objects presented here, there are more stars with pronounced period changes that deserve more detailed scrutiny in the future. LX Cyg (Zijlstra et al. 2004) is a particularly interesting example, which is classified as SC spectral type, just as BH Cru was before its spectrum started to change. Assuming that the $s$ process enrichment in LX Cyg is intrinsic, this star would similarly be placed on the TP-AGB. A period change caused by a recent TP seems plausible. Furthermore, TT Cen (Whitelock 1999), S Sex (Merchán Benítez \& Jurado Vargas 2000), as well as the remarkable objects mentioned in Templeton et al. (2005, R Cen, Z Tau, Y Per) deserve a closer look with high-resolution spectroscopy, which we have to postpone to a future project.

Acknowledgements. The Hermes project is a collaboration between the KU Leuven, the Université Libre de Bruxelles, and the Royal Observatory of Belgium with contributions from the Observatoire de Genève (Switzerland) and the Thüringer Landessternwarte Tautenburg (Germany). Hermes is funded by the Fund for Scientific Research of Flanders (FWO) under the grant G.0472.04, from the Research Council of KU Leuven under grant GST-B4443, from the Fonds National de la Recherche Scientifique under contracts IISN4.4506.05 and FRFC 2.4533.09, and financial support from Lotto (2004) assigned to the Royal Observatory of Belgium. The authors wish to acknowledge the Geneva Observatory and its staff for the generous time allocation on the Swiss Leonhard Euler telescope. We wish to thank the Hermes and Coralie observers who carried out the observations used for this research: P. Beck, P. Degroote, C. Gielen, R. Østensen, W. Pessemier, and T. Verhoelst. S.U. acknowledges support from the Fund for Scientific Research of Flanders (FWO) under grant number G.0470.07, and from the Austrian Science Fund (FWF) under project number P 22911-N16, F.K. from P 18939-N16. This publication makes use of data products from the Two Micron All Sky Survey, which is a joint project of the University of Massachusetts and the Infrared Processing and Analysis Center/California Institute of Technology, funded by the National Aeronautics and Space Administration and the National Science Foundation. We acknowledge with thanks the variable star observations from the AAVSO International Database contributed by observers worldwide and used in this research. We thank the anonymous referee for constructive comments, which helped to improve the paper.

\section{References}

Abia, C., \& Wallerstein, G. 1998, MNRAS, 293, 89

Aringer, B., Girardi, L., Nowotny, W., et al. 2009, A\&A, 503, 913

Busso, M., Gallino, R., \& Wasserburg, G. J. 1999, ARA\&A, 37, 239

Cameron, A. G. W., \& Fowler, W. A. 1971, ApJ, 164, 111

Carpenter, J. M. 2001, AJ, 121, 2851

Catchpole, R. M., \& Feast, M. W. 1971, MNRAS, 154, 197

Catchpole, R. M., Robertson, B. S. C., Lloyd Evans, T. H. H., et al. 1979, SAAOC, 1, 61

Davis, D. N. 1947, ApJ, 106, 28

Fluks, M. A., Plez, B., The, P. S., et al. 1994, A\&AS, 105, 311

Fouqué, P., Le Bertre, T., Epchtein, N., Gugliemo, F., \& Kerschbaum, F. 1992, A\&AS, 93, 151

García-Hernández, D. A., García-Lario, P., Plez, B., et al. 2006, Science, 314, 1751

García-Hernández, D. A., García-Lario, P., Plez, B., et al. 2007, A\&A, 462, 711

Guandalini, R., \& Busso, M. 2008, A\&A, 488, 675

Hron, J. 1991, A\&A, 252, 583

Jura, M., \& Kleinmann, S. G. 1992, ApJS, 79, 105

Karakas, A. 2003, Ph.D. Thesis, Monash University, Australia

Karakas, A. I., Campbell, S. W., \& Stancliffe, R. J. 2010, ApJ, 713, 374

Keenan, P. C., \& Boeshaar, P. C. 1980, ApJS, 43, 379

Kerschbaum, F., \& Hron, J. 1992, A\&A, 263, 97

Kerschbaum, F., \& Hron, J. 1994, A\&AS, 106, 397

Kerschbaum, F., Lebzelter, T., \& Mekul, L. 2010, A\&A, 524, A87

Kipper, T., \& Wallerstein, G. 1990, PASA, 102, 574

Lambert, D. L., Gustafsson, B., Eriksson, K., \& Hinkle, K. H. 1986, ApJS, 62, 373

Lebzelter, T., \& Hron, J. 1999, A\&A, 351, 533

Lebzelter, T., \& Wood, P. R. 2005, A\&A, 441, 1117

Lebzelter, T., \& Wood, P. R. 2007, A\&A, 475, 643

Lebzelter, T., Hinkle, K. H., Wood, P. R., et al. 2005, A\&A, 431, 623 
S. Uttenthaler et al.: The evolutionary state of Miras with changing pulsation periods

Lederer, M. T., Lebzelter, T., Cristallo, S., et al. 2009, A\&A, 502, 913

Lenz, P., \& Breger, M. 2005, Comm. Asteroseism., 146, 53

Little-Marenin, I. R., \& Little, S. J. 1979, AJ, 84, 1374

Little, S. J., Little-Marenin, I. R., \& Hagen-Bauer, W. 1987, AJ, 94, 981

Lloyd Evans, T. 1985, The evolutionary connection between S and C stars -

Evidence from star clusters and the Magellanic Clouds, in Cool Stars with

Excesses of Heavy Elements, ed. M. Jaschek, \& P. C. Keenan (Dordrecht:

D. Reidel Publishing Co.), 163

Loidl, R., Lançon, A., \& Jørgensen, U. G. 2001, A\&A, 371, 1065

Mattei, J. A., \& Foster, G. 1995, JAAVSO, 23, 106

McSaveney, J. A., Wood, P. R., Scholz, M., et al. 2007, MNRAS, 378, 1089

Mennessier, M. O., Mowlavi, N., Alvarez, R., \& Luri, X. 2001, A\&A, 374, 968

Merchán Benítez, P., \& Jurado Vargas, M. 2000, A\&A, 353, 264

Merrill, P. W. 1952, ApJ, 116, 18

Merrill, P. W., Deutsch, A. J., \& Keenan, P. C. 1962, ApJ, 136, 21

Nowotny, W., Höfner, S., \& Aringer, B. 2010, A\&A, 514, A35

Pourbaix, D., Platais, I., Detournay, S., et al. 2003, A\&A, 399, 1167

Raskin, G., Van Winckel, H., Hensberge, H., et al. 2010, A\&A, 526, A69

Riebel, D., Meixner, M., Fraser, O., et al. 2010, ApJ, 723, 1195

Sackmann, I. J., \& Boothroyd, A. I. 1992, ApJ, 392, L71

Samus, N. N., Durlevich, O. V., et al. 2009, General Catalogue of Variable Stars (Samus+ 2007-2010), VizieR On-line Data Catalog

Schwarzschild, M., \& Härm, R. 1965, ApJ, 142, 855
Skiff, B. A. 2010, Catalogue of Stellar Spectral Classifications, VizieR On-line Data Catalog

Skrutskie, M. F., Cutri, R. M., Stiening, S., et al. 2006, AJ, 131, 1163

Smith, V. V., Plez, B., Lambert, D. L., \& Lubowich, D. A. 1995, ApJ, 441, 735

Szatmáry, K., Kiss, L. L., \& Bebesi, Zs. 2003, A\&A, 398, 277

Templeton, M. R., Mattei, J. A., \& Willson, L. A. 2005, AJ, 130, 776

Templeton, M. R., Willson, L. A., \& Foster, G. 2008, JAAVSO, 36, 1

Uttenthaler, S., \& Lebzelter, T. 2010, A\&A, 510, A62

Uttenthaler, S., Hron, J., Lebzelter, T., et al. 2007a, A\&A, 463, 251

Uttenthaler, S., Lebzelter, T., Palmerini, S., et al. 2007b, A\&A, 471, L41

Van Eck, S., \& Jorissen, A. 1999, A\&A, 345, 127

Vanture, A. D., Wallerstein, G., Brown, J. A., \& Bazan, G. 1991, ApJ, 381, 278

Vanture, A. D., Smith, V. V., Lutz, J., et al. 2007, PASP, 119, 147

Whitelock, P. A., 1999, New Astron. Rev., 43, 437

Whitelock, P. A., Feast, M. W., Marang, F., \& Groenewegen, M. A. T. 2006, MNRAS, 369, 751

Wood, P. R. 1975, in Multiple Periodic Variable Stars, ed. W. S. Fitch (Dordrecht: Reidel), IAU Coll., 29, 69

Wood, P. R., \& Zarro, D. M. 1981, ApJ, 247, 247

Wood, P. R., Alcock, C., Allsman, R. A., et al. 1991, IAUS, 191, 151

Ya'ari, A., \& Tuchman, Y. 1996, ApJ, 456, 350

Zijlstra, A. A., \& Bedding, T. R. 2002, JAAVSO, 31, 2

Zijlstra, A. A., Bedding, T. R., Markwick, A. J., et al. 2004, MNRAS, 352, 325 